\title{
Tufts PACE Clinical Predictive Model Registry: update 1990 through 2015
}

Benjamin S. Wessler ${ }^{1,2}$, Jessica Paulus ${ }^{2}$, Christine M. Lundquist ${ }^{2}$, Muhammad Ajlan ${ }^{2,3}$, Zuhair Natto², William A. Janes², Nitin Jethmalani², Gowri Raman ${ }^{4}$, Jennifer S. Lutz ${ }^{2}$ and David M. Kent ${ }^{2 *}$

\begin{abstract}
Background: Clinical predictive models (CPMs) estimate the probability of clinical outcomes and hold the potential to improve decision-making and individualize care. The Tufts Predictive Analytics and Comparative Effectiveness (PACE) CPM Registry is a comprehensive database of cardiovascular disease (CVD) CPMs. The Registry was last updated in 2012, and there continues to be substantial growth in the number of available CPMs.
\end{abstract}

Methods: We updated a systematic review of CPMs for CVD to include articles published from January 1990 to March 2015. CVD includes coronary artery disease (CAD), congestive heart failure (CHF), arrhythmias, stroke, venous thromboembolism (VTE), and peripheral vascular disease (PVD). The updated Registry characterizes CPMs based on population under study, model performance, covariates, and predicted outcomes.

Results: The Registry includes 747 articles presenting 1083 models, including both prognostic $(n=1060)$ and diagnostic $(n=23)$ CPMs representing 183 distinct index condition/outcome pairs. There was a threefold increase in the number of CPMs published between 2005 and 2014, compared to the prior 10-year interval from 1995 to 2004. The majority of CPMs were derived from either North American $(n=455,42 \%)$ or European $(n=344,32 \%)$ populations. The database contains 265 CPMs predicting outcomes for patients with coronary artery disease, 196 CPMs for population samples at risk for incident CVD, and 158 models for patients with stroke. Approximately two thirds $(n=701,65 \%)$ of CPMs report a c-statistic, with a median reported c-statistic of 0.77 (IQR, 0.05). Of the CPMs reporting validations, only $333(57 \%)$ report some measure of model calibration. Reporting of discrimination but not calibration is improving over time ( $p$ for trend $<0.0001$ and 0.39 respectively).

Conclusions: There is substantial redundancy of CPMs for a wide spectrum of CVD conditions. While the number of CPMs continues to increase, model performance is often inadequately reported and calibration is infrequently assessed. More work is needed to understand the potential impact of this literature.

Keywords: Prediction, Cardiovascular disease risk factors, Cerebrovascular disease/stroke, Modeling, Prognostic factor, Clinical predictive model, Coronary artery disease, Risk stratification, Methods

\section{Background}

Prognosis is an essential task in clinical practice, yet it is a task in which physicians have repeatedly demonstrated poor performance [1]. Clinical prediction models (CPMs), empirically derived from large databases using mathematical models, have been developed to provide objective, patientspecific risk estimates of the probability of important

\footnotetext{
* Correspondence: dkent1@tuftsmedicalcenter.org

${ }^{2}$ Predictive Analytics and Comparative Effectiveness (PACE) Center, Institute for Clinical Research and Health Policy Studies (ICRHPS), Tufts Medical Center/Tufts University School of Medicine, 800 Washington Street, Box 63, Boston, MA 02111, USA

Full list of author information is available at the end of the article
}

outcomes based on easily ascertained clinical variables. These tools are designed to enable clinicians to "personalize" risk-sensitive medical decisions for individual patients [2]. In cardiovascular disease (CVD) and other fields of medicine, CPMs are now incorporated into numerous clinical practice guidelines [3-5].

Despite the compelling rationale for CPMs, as well as their growth in the literature [6] and increasing incorporation into clinical guidelines, application of these models remains limited and their potential impact on clinical care remains largely unknown. There have been recent collaborative efforts to understand the extent and 
limitations of the CPM literature, to establish methodological standards [7] and reporting guidelines [8], and to create a community of researchers dedicated to advancing this field, including the creation of a new journal [9]. To contribute to this broad effort to better understand the scope and limitations of the literature, we created the Tufts Predictive Analytics and Comparative Effectiveness (PACE) Clinical Predictive Model (CPM) Registry, which describes published CPMs for patients at risk for and with known CVD. Herein, we report an update to this field synopsis and present an online version of the registry.

The Registry is available at http://pace.tuftsmedical center.org/cpm to aid clinicians and researchers in understanding the state of CPM development across the spectrum of CVD.

\section{Methods}

\section{Study search and selection}

Our search strategy has been previously described [6]. Briefly, we performed a PubMed search for Englishlanguage articles containing newly developed CPMs. A CPM was defined as a model that provides a method to calculate or categorize an individuals' risk for a binary outcome. Here, we updated our search to include articles published through March 31, 2015. We supplemented this search by scanning reference lists to ensure completeness of the database (Fig. 1). CVD includes coronary artery disease (CAD), congestive heart failure (CHF), arrhythmias, stroke, venous thromboembolism (VTE), and peripheral vascular disease (PVD).
For this registry, a CPM is defined as a predictive model that can be used to estimate an individual patient's absolute risk for a binary outcome. Detailed inclusion and exclusion criteria have been previously reported [6]. The Registry includes articles that describe both prognostic and diagnostic models. Included articles describe CPMs for patients at risk of developing CVD and also for patients with known CVD that predict binary outcomes (e.g., MI, death, or composite endpoints). To be included in the database, articles must include sufficient information about the CPM-typically in the form of a point score, equation, decision tree, nomogram, or online calculator-for readers to be able to generate individual predictions.

\section{Data extraction}

This report focuses on de novo CPMs, defined as newly derived CPMs. We extracted CPM information directly into our Microsoft Access 2010 database. Blinded double extractions of key fields were done to ensure consistency of extracted data. Discrepancies were discussed to arrive at a consensus.

We extracted information at the article and model level. All data extraction done after 2015 has been aligned with the recently published Transparent Reporting of a Multivariable Prediction Model for Individual Prognosis or Diagnosis (TRIPOD) statement [8] and Critical Appraisal and Data Extraction for Systematic Reviews of Prediction Modeling Studies (CHARMS) Checklist [10]. Measures of CPM discrimination and calibration were extracted as well as information on the type of validation reported at the time of publication. CPMs were characterized based on index condition/outcome pairs (I/O pairs). CPMs focused

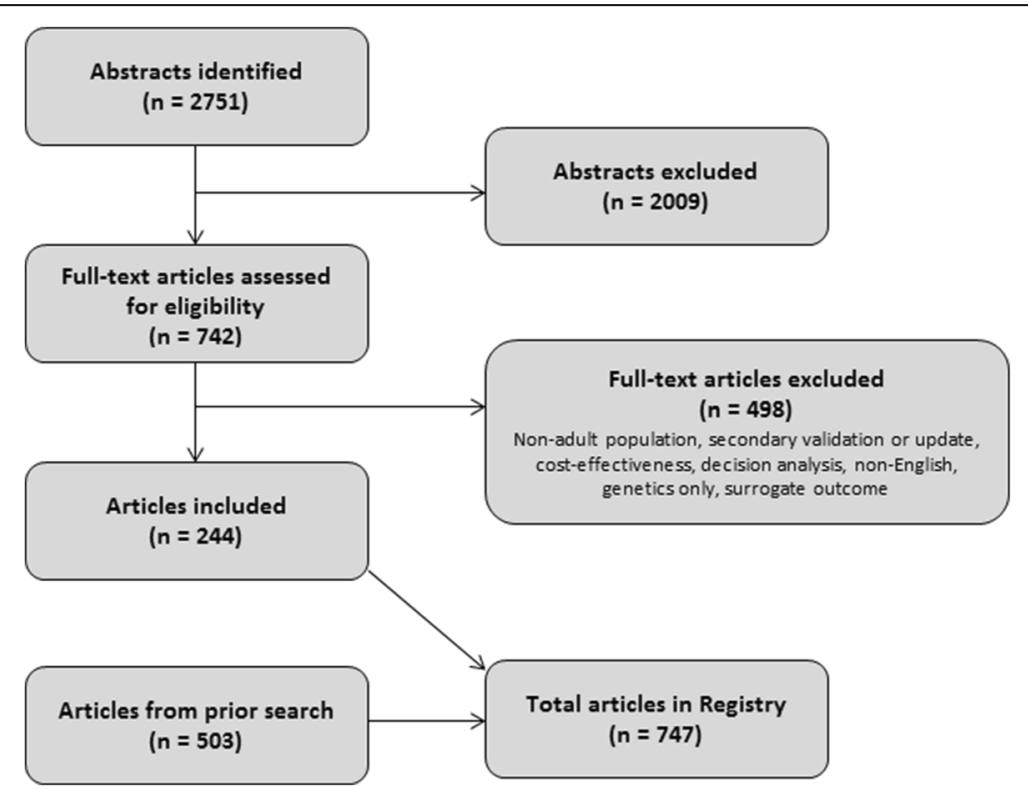

Fig. 1 PubMed was searched for relevant articles from 1990 to March 2015 
on predicting the development of incident CVD were characterized as "population sample" CPMs. Outcomes were categorized as mortality, major adverse cardiac events (MACE), major adverse cardiovascular or cerebrovascular events (MACCE), other composite outcomes, or other clinical events. We created a clinically oriented classification scheme to describe CPM variables based on Medical Subject Headings (MeSH) terms. Where an appropriate $\mathrm{MeSH}$ term was not identified, we categorized covariates with an appropriate heading. We present the most common covariates in the Registry (overall) and also across the top 5 index conditions.

We examined secular trends in the reporting of CPM performance. We compared the proportion of models reporting discrimination, some measure of calibration, and inclusion of a web- or computer-based calculator for estimating probabilities across 5-year intervals from 1990 to 2015 using a chi-square test for trend.

\section{Results}

\section{Overall registry}

The Registry includes 747 articles presenting prognostic $(n=1060)$ and diagnostic $(n=23)$ CPMs (Fig. 1). Three hundred seventy-four CPMs were added to the Registry during this update. There was a threefold increase in the number of CPMs published between 2005 and 2014 (the last full decade for which we have data), compared to the prior 10-year interval (1995-2004) (Fig. 2). CPMs were most commonly published in specialty journals (Table 1). Circulation published 53 (7.1\%) and the Journal of American College of Cardiology published 45 (6.0\%) of the articles included in the Registry. The majority of CPMs were derived from either North American $(n=455$, $42 \%)$ or European $(n=344,32 \%)$ populations (Fig. 3). The most common statistical method used to create CPMs
Table 1 Journals ranked by number of CPMs published in 1990-2015

\begin{tabular}{lll}
\hline Journal & Count & Rank \\
\hline Circulation & 53 & 1 \\
Journal of the American College of Cardiology & 45 & 2 \\
Stroke & 40 & 3 \\
American Journal of Cardiology & 36 & 4 \\
Annals of Thoracic Surgery & 25 & 5 \\
European Heart Journal & 21 & 6 \\
American Heart Journal & 21 & 7 \\
International Journal of Cardiology & 17 & 8 \\
Journal of Vascular Surgery & 16 & 9 \\
Journal of the American Medical Association & 15 & 10 \\
Other & 455 & \\
Total & 747 & \\
\hline
\end{tabular}

Journals ranked according to number of published CPM articles from 1990 to March 2015. "Other" includes all other journals publishing CPM reports. CPM indicates clinical predictive model

was logistic regression (55\%), followed by Cox regression (33\%). CPMs were derived from a variety of data sources: 63\% derived CPMs from cohort studies, 19\% used registry data, and 10\% used RCT data. For the top 10 index conditions, CPMs most commonly (51\%) predicted mortality over a short time frame $(<3$ months). Forty-four percent of these CPMs predicted mortality over a long time frame (> 6 months).

For the 10 most commonly studied I/O pairs, 102 models (18\%) did not report the number of events in the derivation cohort (Table 2). For the top ten I/O pair de novo CPMs that reported the number of events, there was a wide range of events per included variable (EPV) $($ median $=25$ [IQR, 12]).

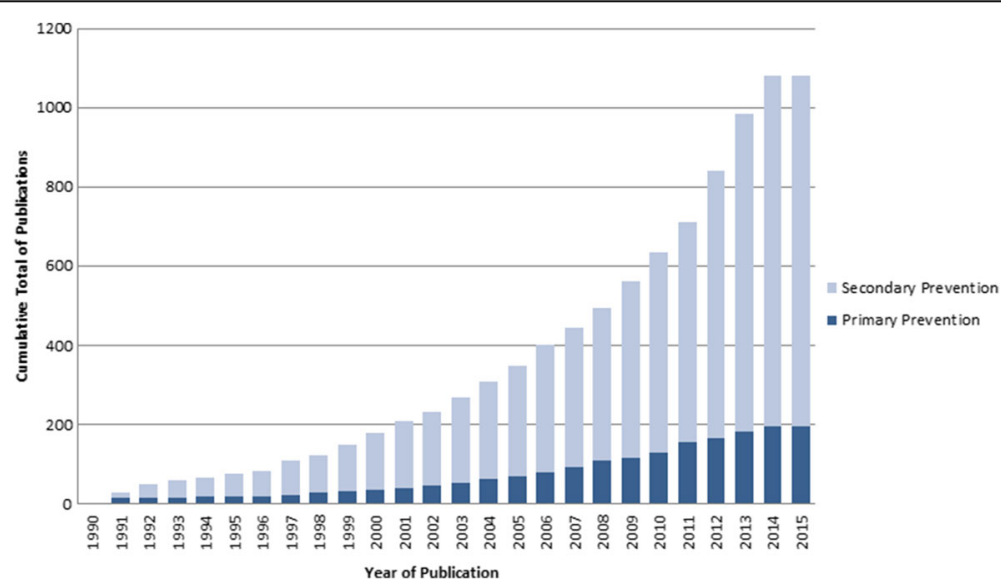

Fig. 2 Cumulative growth in published CPM articles included in the Tufts CPM database over time (January 1990-March 2015). Dark blue represents models derived on CVD-free population samples. Light blue represents models derived on patients with specific cardiovascular conditions at baseline 


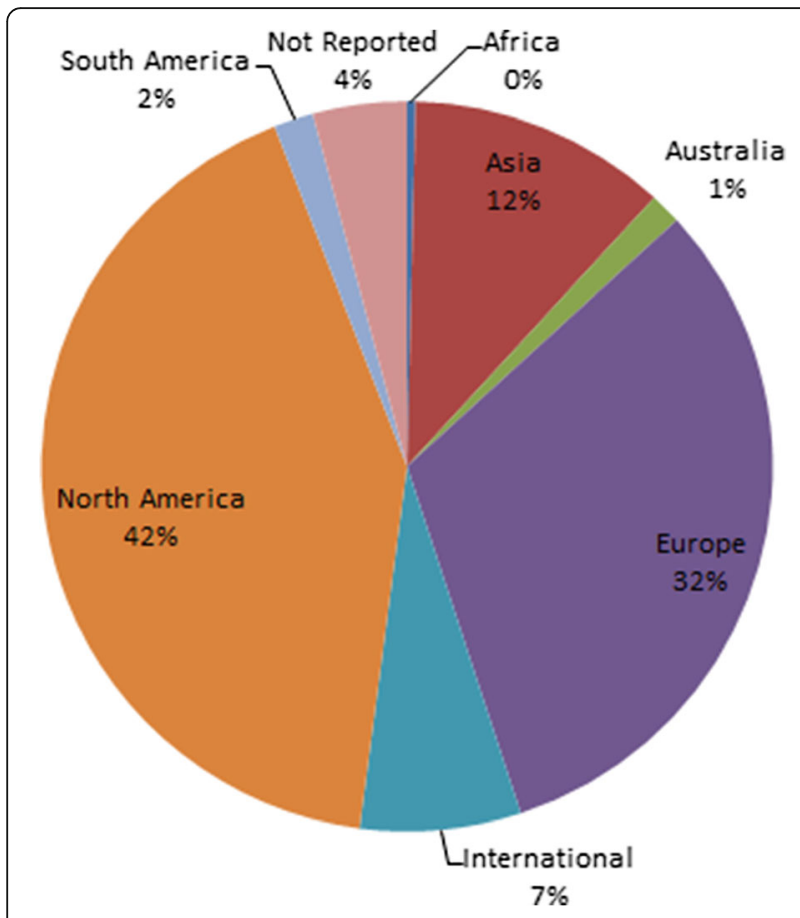

Fig. 3 CPMs by derivation cohort geographic region

\section{Clinical focus}

These CPMs represent 24 index conditions and 183 unique $\mathrm{I} / \mathrm{O}$ pairs. The 10 most frequently studied index conditions and associated summary discriminatory performance are shown in Fig. 4. There are 265 CPMs for patients with known coronary artery disease (CAD), 196 for population samples, and 158 for patients with prior stroke. The diagnostic CPMs included in this database most commonly predict the presence or absence of venous thromboembolic disease (six models) and CAD (four models). Overall, the most commonly predicted outcome was mortality (40\%), followed by MACE (9\%), stroke (6\%), functional outcomes (6\%), and MACCE (6\%). CPMs predicting mortality were most commonly published for patients with known CAD (126 models), followed by heart failure (100 models) and stroke (51 models). CPMs predicting composite outcomes representing MACE were most frequently developed for population samples (42 models), followed by patients with CAD (27 models) and chest pain (16 models).

\section{Covariate environment}

Covariate frequencies are shown in Fig. 5. Across the entire Registry, 798 (74\%) of the CPMs include a variable for age. CPMs frequently included covariates representing renal function (34\%), blood pressure (33\%), CAD/MI (27\%), and diabetes (32\%). The common covariates are shown for common index conditions. Only 6\% of CPMs in this Registry include a covariate for race (counts include interaction terms).

\section{Model performance}

Of the 1083 CPMs in the Registry, 701 (65\%) report a $c$-statistic. Discrimination was more frequently reported in logistic regression-based models $(455 / 612,74 \%)$ than in Cox regression models (190/357, 53\%). The median reported $c$-statistic was 0.77 (IQR, 0.05). Of the 10 most common index conditions, discrimination was highest for CPMs predicting outcomes following stroke (106/ 158 reporting, median c-statistic 0.82 [IQR, 0.04]) and cardiac arrest (17/27 reporting, median $c$-statistic 0.83 [IQR, 0.02]) (Fig. 4). Discrimination was lowest for CPMs predicting outcomes for patients with valve disease (17/22 reporting, median $c$-statistic 0.72 [IQR 0.04]). Forty percent $(n=433)$ of CPMs describe an internal validation exercise (including testing on random and non-random subset of the same overall cohort) and $25 \%(n=274)$

Table 2 Index condition/outcome (I/O) pairs of de novo models

\begin{tabular}{lllll}
\hline //O pair & Models reporting events & Variables per model & Events per model & Events per variable (EPV) \\
\hline CAD-mortality & $102(81 \%)$ & $9(6-12)$ & $233(125-709)$ & $35(15-64)$ \\
CHF-mortality & $80(80 \%)$ & $7(5-9)$ & $131(81-253)$ & $24(13-32)$ \\
Population sample-MACE/MACCE & $53(74 \%)$ & $7(6-8)$ & $312(137-686)$ & $36(20-100)$ \\
Stroke-functional outcome & $48(92 \%)$ & $6(4-8)$ & $114(43-310)$ & $16(9-44)$ \\
Stroke-mortality & $41(80 \%)$ & $5(4-6)$ & $72(40-174)$ & $15(12-42)$ \\
CAD-MACE/MACCE & $43(88 \%)$ & $6(4-9)$ & $143(68-254)$ & $21(13-39)$ \\
Cardiac surgery-mortality & $31(97 \%)$ & $10(7-13)$ & $171(95-295)$ & $21(12-31)$ \\
Population sample-mortality & $22(73 \%)$ & $5(5-7)$ & $377(116-1716)$ & $48(19-343)$ \\
Population sample-stroke & $18(69 \%)$ & $6(5-8)$ & $227(112-309)$ & $30(20-52)$ \\
Aortic disease-mortality & $23(92 \%)$ & $4(3-7)$ & $43(26-136)$ & $14(5-23)$
\end{tabular}

Numbers reported are $n$ (\%) or median (IQR). Top 10 index condition/outcome (I/O) pairs. We report here variables included in the model (as opposed to candidate variables).

$C A D$ coronary artery disease, $C H F$ congestive heart failure, $M A C E$, major adverse cardiovascular events, MACCE major adverse cardiovascular and cerebrovascular events 


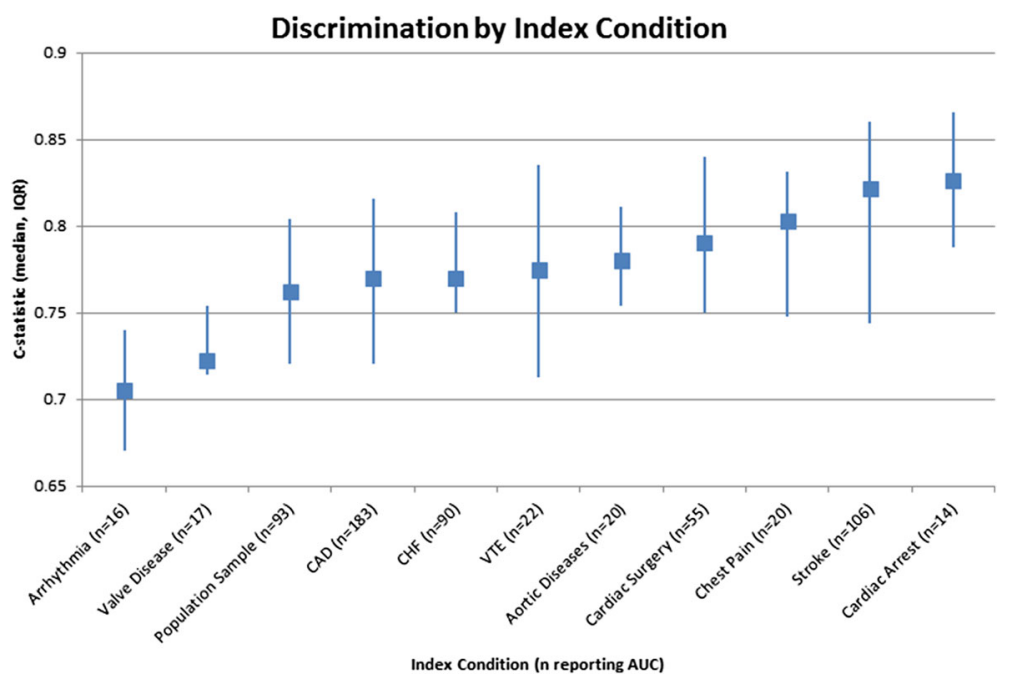

Fig. 4 Discrimination of CPMs by index condition. Discrimination is reported as C-statistic (median, IQR). CPM, clinical prediction model; CAD, coronary artery disease; CHF, congestive heart failure; VTE, venous thromboembolism

report validation on a population sample separate from the derivation sample. (This does not include external validations published separately.) Of the CPMs included in this Registry reporting some type of validation $(n=577)$, only 333 (57\%) report some measure of model calibration. For the reports presenting an external validation, only $50 \%$ report calibration. Of the CPMs reporting calibration that were published after May 2012, only 93 (56\%) report a Hosmer-Lemeshow statistic.

\section{Time trends}

The frequency of reporting CPM discrimination as part of the original CPM description increased from 1990 to
2015 ( $p$ for trend $<0.001$ ). It is also increasingly common to see calculators presented alongside CPMs to enhance clinical use ( $p$ for trend $<0.01)$. There is no change over time in the frequency of reporting CPM calibration ( $p$ for trend $=0.39)($ Table 3$)$.

\section{Website}

The Tufts PACE CPM Registry is publically available at http://pace.tuftsmedicalcenter.org/cpm. CPMs are searchable by PubMed ID, index condition, outcome, and Medical Subject Headings (MeSH) terms. Screenshots are shown in the Additional file 1. Extracted information, including sample size, number of events, follow-up duration, and

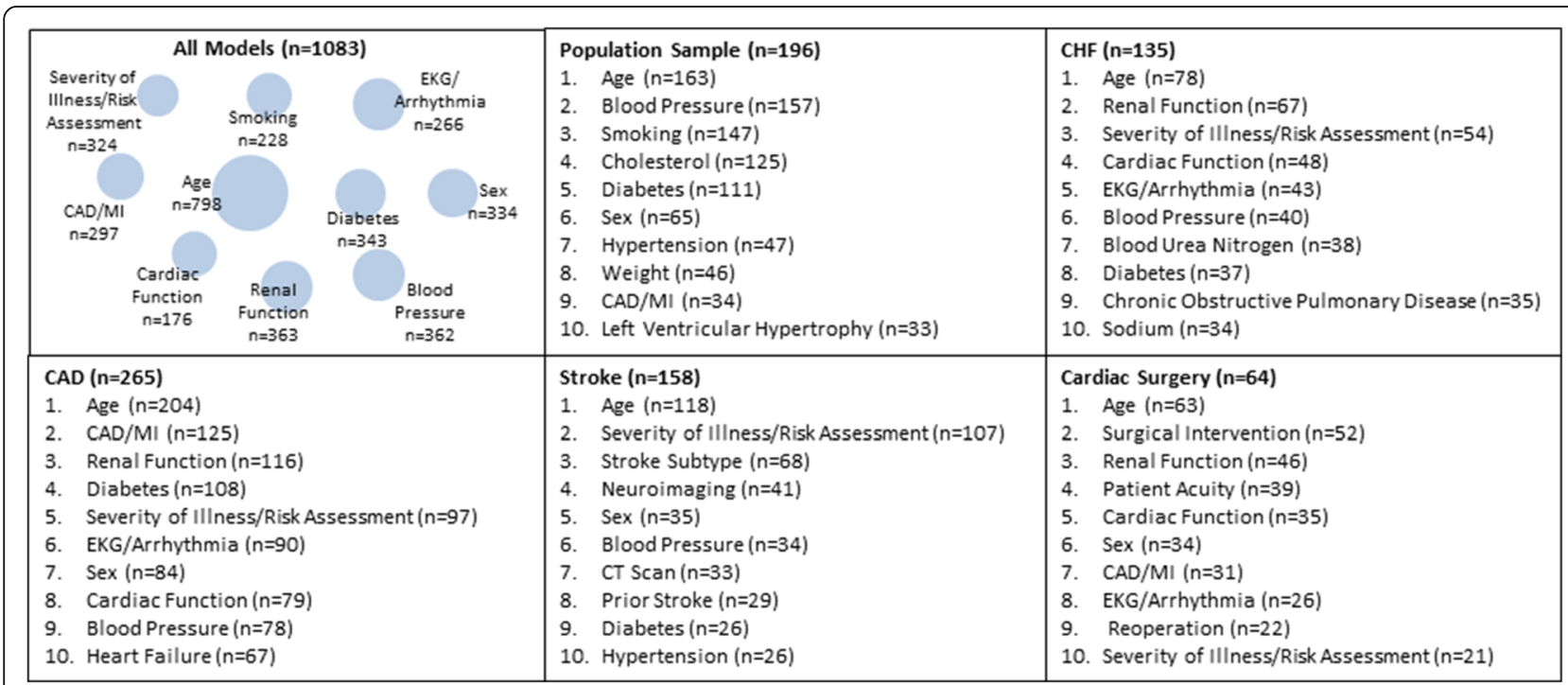

Fig. 5 Frequency of covariate categories among all covariates $(n=9641)$ in the Tufts PACE CPM Registry. Top covariates across the top 5 index conditions are also presented 
Table 3 Time trends for reporting discrimination and calibration and providing a calculator

\begin{tabular}{|c|c|c|c|c|c|c|c|}
\hline \multirow{2}{*}{$\begin{array}{l}\text { Time } \\
\text { period }\end{array}$} & \multirow{2}{*}{$\begin{array}{l}\text { Total } \\
\text { models }(n)\end{array}$} & \multicolumn{2}{|l|}{ Discrimination } & \multicolumn{2}{|l|}{ Calibration } & \multicolumn{2}{|l|}{ Calculator } \\
\hline & & Reporting AUC (\%) & $p$ for trend & Reporting calibration (\%) & $p$ for trend & Providing calculator (\%) & $p$ for trend \\
\hline 1990-1995 & 75 & 31 & $<0.0001$ & 58 & 0.39 & 0 & $<0.01$ \\
\hline 1996-2000 & 102 & 49 & & 48 & & 0 & \\
\hline $2001-2005$ & 171 & 61 & & 53 & & 1 & \\
\hline $2006-2010$ & 285 & 72 & & 65 & & 3 & \\
\hline $2011-2015$ & 450 & 71 & & 57 & & 4 & \\
\hline
\end{tabular}

measures of statistical performance, is presented at the model level.

\section{Discussion}

Here, we report updated results from the Tufts PACE CPM Registry and introduce the publically available website, which can serve as a resource to help understand major trends in CPM development. This registry documents continued and accelerating growth of CVD CPMs and an important trend of improved reporting of statistical performance over time. Nevertheless, calibration remains poorly reported (less than $40 \%$ of all CPMs and in only 57\% of CPMs reporting on validation), despite methodological work emphasizing the critical importance of calibration for decision-making [11].

Perhaps, our most striking finding is the number and continued growth of CPMs in CVD, despite substantial apparent redundancy of models. This growth in the literature likely reflects the increasing ease with which these models can be developed. With the growing volume of and access to research and clinical databases, in addition to the broad availability of software packages, barriers to developing new models are rapidly diminishing. Nevertheless, barriers to clinical translation remain. These barriers are incompletely understood but go well beyond the methodological and statistical issues addressed by prior guidelines $[7,12]$. We believe barriers to dissemination relate to whether the rationale for a CPM is strongly linked to a specific decisional context (i.e., if they inform critical decisions), whether its output is informative from a decision analytic perspective [13], whether its output leverages into clinicians' and patients' natural decisionmaking process [14], and how it fits into the highly demanding and sometimes chaotic clinical workflow [15]. While this study did not include any evaluation of the dissemination of CPMs into practice or their influence on clinical decision-making, we suspect that the growth in the number of reported CPMs was not accompanied by a commensurate increase in their use in clinical practice. If CPMs are to deliver on the promise of supporting more individualized evidence-based decisions, better understanding this gap remains an important challenge.

We have created a publically available registry for the research community. Sample screenshots are shown in
Additional file 1 and present summary data about each CPM. CPMs are easily searchable by index condition, author, and PubMed ID, and information about model development and performance are readily assessed. Ideally, this Registry will be leveraged when future CPM building is considered to confirm if there is a clinical need for a new CPM and to help identify established predictors of outcomes. The Registry might be consulted by reviewers and editors when evaluating the scientific and clinical merits of new models. The Registry also permits not only the study of CPMs within specific index conditions and outcomes, but also the study of predictor variables of interest across different index conditions [16, 17].

The Prognosis Research Strategy (PROGRESS) group $[12,18]$ has outlined standards for creating predictive models, and the Transparent Reporting of a Multivariable Model for Individual Prognosis or Diagnosis (TRIPOD) statement [8] has highlighted reporting standards that are increasingly being adopted by journals across a number of disciplines. These important efforts arise from welldocumented gaps in reporting $[19,20]$. In our systematic review, many more Cox models than logistic models were excluded from the Registry since, in accordance with our original inclusion criteria; many Cox models did not describe the baseline hazard or provide an alternative way to generate individual patient predictions and thus were excluded. Since 2012, 46\% of full-text articles that are screened for inclusion are excluded from our Registry because they do report a usable model. In our registry, CPMs remain incompletely reported with relatively little focus on model calibration, despite the important role of good calibration in preventing harmful prediction models $[11,21]$. While reporting remains incomplete, we note marked improvements in reporting of discrimination (c-statistic) over time. We also document that it is increasingly common to find calculators for bedside use presented alongside CPMs. These reporting observations are notes of optimism for a field that has struggled with reporting consistency.

The final covariates included in the CPMs in this Registry show the importance of common cardiovascular risk factors in predicting outcomes for patients at risk and with known CVD. Renal function is a common covariate across the entire database; however, it is interesting to 
note that it is less frequently seen in CPMs predicting incident CVD (population sample CPMs) and also CPMs predicting outcomes after stroke. This may be related to the time horizon of prediction or the severity of the index condition. We have previously described that sex is more often included for CPMs predicting incident CVD compared to CPMs for diseased populations [22], an observation that may be partially attributed to the presence of index event bias [23], which may tend to diminish the apparent effects of risk factors among patients selected for the presence of an index event or condition. Ongoing efforts seek to describe these variables at a more granular level and to describe the directionality and effect size of a number of common predictors across various $\mathrm{I} / \mathrm{O}$ pairs.

Our registry has several limitations. Because there is no MeSH term to identify predictive models, our search strategy may have missed some CPMs that met the inclusion criteria. We are continuing to add CPMs as they are discovered in the course of enhancing the database. Additionally, the registry excluded articles reporting CPMs if they did not provide a means to calculate a prediction (e.g., the authors reported odds ratios but no intercept). Given the level of detailed extraction required to populate the web-based resource and the continued rapid expansion of CPMs, our updated registry now requires further updating. The registry does not focus on CPM validations, so at this time, the performance of these models, outside of the derivation datasets, is generally unknown.

\section{Conclusion}

The Tufts PACE CPM Registry (available at http://pace. tuftsmedicalcenter.org/cpm) is a publically available resource of CVD CPMs. This Registry documents substantial redundancy of CPMs for a wide spectrum of CVD conditions. Model performance is often inadequately reported, though discrimination (but not calibration) reporting appears to be improving over time. More work is needed to understand the potential impact of this literature.

\section{Additional file}

Additional file 1: Website screenshots of Tufts PACE CPM Registry (Accessed 6/4/2017). (DOCX $682 \mathrm{~kb}$ )

\section{Acknowledgements}

Not applicable.

\section{Funding}

This work was partially supported through two Patient-Centered Outcomes Research Institute (PCORI) grants (Pilot Project Program Award [IP2PI000722] and Methods Award [ME-1606-35555]), as well as by the National Institutes of Health (U01NS086294, T32HL069770, UL1 TR001064).

\section{Availability of data and materials}

The datasets generated and/or analyzed during the current study are partially available at the Tufts PACE CPM Registry website, http:// pace.tuftsmedicalcenter.org/cpm. Additional data elements from the registry may be available from the corresponding author upon reasonable request.

\section{Disclosures}

All statements in this report, including its findings and conclusions, are solely those of the authors and do not necessarily represent the views of the Patient-Centered Outcomes Research Institute (PCORI), its Board of Governors, or Methodology Committee.

\section{Authors' contributions}

BW and DK made substantial contributions to the conception and design, the acquisition of the data, or the analysis and interpretation of the data. BW and DK had been involved in drafting the manuscript or revising it critically for important intellectual content. BW, JP, CL, MA, ZN, NJ, WJ, GR, JL, and DK have participated sufficiently in the work to take public responsibility for appropriate portions of the content, agreed to be accountable for all aspects of the work in ensuring that questions related to the accuracy or integrity of any part of the work are appropriately investigated and resolved, and have read and given final approval of the version to be published. NJ has participated sufficiently in the work to take public responsibility for appropriate portions of the contect, agreed to be accountable for all aspects of the work in ensuring that questions related to the accuracy or integrity of any part of the work are appropriately investigate and resolved, and has read and given final approval of the version to be published.

Ethics approval and consent to participate Not applicable.

\section{Consent for publication}

Not applicable.

\section{Competing interests}

The authors declare that they have no competing interests.

\section{Publisher's Note}

Springer Nature remains neutral with regard to jurisdictional claims in published maps and institutional affiliations.

\section{Author details}

${ }^{1}$ Division of Cardiology, Tufts Medical Center, Boston, USA. ${ }^{2}$ Predictive Analytics and Comparative Effectiveness (PACE) Center, Institute for Clinical Research and Health Policy Studies (ICRHPS), Tufts Medical Center/Tufts University School of Medicine, 800 Washington Street, Box 63, Boston, MA 02111, USA. ${ }^{3}$ King Abdulaziz Cardiac Center, King Abdulaziz Medical City (Riyadh), Ministry of National Guard - Health Affairs, Riyadh, Kingdom of Saudi Arabia. ${ }^{4}$ Center for Clinical Evidence Synthesis, ICRHPS, Medical Center/ Tufts University School of Medicine, Boston, USA.

Received: 15 June 2017 Accepted: 23 November 2017

Published online: 21 December 2017

\section{References}

1. Christakis NA, Lamont EB. Extent and determinants of error in doctors' prognoses in terminally ill patients: prospective cohort study. BMJ. 2000;320:469-72.

2. Moons KGM, Altman DG, Vergouwe $Y$, Royston P. Prognosis and prognostic research: application and impact of prognostic models in clinical practice. BMJ. 2009;338:b606.

3. Stone NJ, Robinson JG, Lichtenstein AH, Bairey Merz CN, Blum CB, Eckel RH, Goldberg AC, Gordon D, Levy D, Lloyd-Jones DM, McBride P, Schwartz JS, Shero ST, Smith SC, Watson K, Wilson PWF. 2013 ACC/AHA guideline on the treatment of blood cholesterol to reduce atherosclerotic cardiovascular risk in adults: a report of the American College of Cardiology/American Heart Association Task Force on practice guidelines. J Am Coll Cardiol. 2014;63: 2889-934

4. January CT, Wann LS, Alpert JS, Calkins H, Cleveland JC, Cigarroa JE, Conti JB, Ellinor PT, Ezekowitz MD, Field ME, Murray KT, Sacco RL, Stevenson WG, Tchou PJ, Tracy CM, Yancy CW, Cleveland JC, Conti JB, Ellinor PT, Ezekowitz MD, Field ME, Murray KT, Sacco RL, Stevenson WG, Tchou PJ, Tracy CM, 
Yancy CW. 2014 AHA/ACC/HRS guideline for the management of patients with atrial fibrillation: a report of the American College of Cardiology/ American Heart Association Task Force on practice guidelines and the Heart Rhythm Society. J Am Coll Cardiol. 2014:64:e1-e76.

5. Yancy CW, Jessup M, Bozkurt B, Butler J, Casey DE, Drazner MH, Fonarow GC, Geraci SA, Horwich T, Januzzi JL, Johnson MR, Kasper EK, Levy WC, Masoudi FA, McBride PE, McMurray JJV, Mitchell JE, Peterson PN, Riegel B, Sam F, Stevenson LW, Tang WHW, Tsai EJ, Wilkoff BL. 2013 ACCF/AHA guideline for the management of heart failure: a report of the American College of Cardiology Foundation/American Heart Association Task Force on practice guidelines. Circulation. 2013;128:e240-327.

6. Wessler BS, Lai Yh L, Kramer W, Cangelosi M, Raman G, Lutz JS, Kent DM Clinical prediction models for cardiovascular disease: Tufts Predictive Analytics and Comparative Effectiveness Clinical Prediction Model Database. Circ Cardiovasc Qual Outcomes. 2015:8:368-75.

7. Steyerberg EW, Moons KGM, van der Windt DA, Hayden JA, Perel P, Schroter S, Riley RD, Hemingway H, Altman DG. Prognosis Research Strategy (PROGRESS) 3: prognostic model research. PLoS Med. 2013;10:e1001381.

8. Collins GS, Reitsma JB, Altman DG, Moons KGM. Transparent reporting of a multivariable prediction model for individual prognosis or diagnosis (TRIPOD): the TRIPOD statement. Circulation. 2015;131:211-9.

9. Moons KGM, Cook N, Collins G. A new community for those involved and interested in diagnosis and prognosis. Diagnostic Progn Res. 2017;1:5.

10. Moons KGM, de Groot JAH, Bouwmeester W, Vergouwe Y, Mallett S, Altman DG, Reitsma JB, Collins GS. Critical appraisal and data extraction for systematic reviews of prediction modelling studies: the CHARMS checklist. PLoS Med. 2014;11:e1001744

11. Van Calster B, Nieboer D, Vergouwe Y, De Cock B, Pencina MJ, Steyerberg EW. A calibration hierarchy for risk models was defined: from utopia to empirical data. J Clin Epidemiol. 2016;74:167-76.

12. Hemingway H, Croft P, Perel P, Hayden JA, Abrams K, Timmis A, Briggs A, Udumyan R, Moons KGM, Steyerberg EW, Roberts I, Schroter S, Altman DG, Riley RD. Prognosis research strategy (PROGRESS) 1: a framework for researching clinical outcomes. BMJ. 2013;346:e5595.

13. Vickers AJ, Elkin EB. Decision curve analysis: a novel method for evaluating prediction models. Med Decis Mak. 26:565-74.

14. Zikmund-Fisher BJ. The right tool is what they need, not what we have: a taxonomy of appropriate levels of precision in patient risk communication. Med Care Res Rev. 2013;70:37S-49S.

15. Chisholm CD, Collison EK, Nelson DR, Cordell WH. Emergency department workplace interruptions: are emergency physicians "interrupt-driven" and "multitasking"? Acad Emerg Med. 2000;7:1239-43.

16. Paulus JK, Lai LYH, Lundquist C, Daneshmand A, Buettner H, Lutz JS, Raman $G$, Wessler BS, Kent DM. Field synopsis of the role of sex in stroke prediction models. J Am Heart Assoc. 2016;5:1-16.

17. Paulus JK, Wessler BS, Lundquist C, Lai LLY, Raman G, Lutz JS, Kent DM. Field synopsis of sex in clinical prediction models for cardiovascular disease. Circ Cardiovasc Qual Outcomes. 2016;9:S8-S15.

18. Royston P, Moons KGM, Altman DG, Vergouwe Y. Prognosis and prognostic research: developing a prognostic model. BMJ. 2009;338:b604.

19. Collins GS, de Groot JA, Dutton S, Omar O, Shanyinde M, Tajar A, Voysey M, Wharton R, Yu L-M, Moons KG, Altman DG. External validation of multivariable prediction models: a systematic review of methodological conduct and reporting. BMC Med Res Methodol. 2014;14:40.

20. Siontis GCM, Tzoulaki I, Castaldi PJ, loannidis JPA. External validation of new risk prediction models is infrequent and reveals worse prognostic discrimination. J Clin Epidemiol. 2015;68:25-34.

21. Olchanski N, Cohen JT, Neumann PJ, Wong JB, Kent D. Understanding the value of individualized information: the impact of poor calibration or discrimination in outcome prediction models. Med Decis Making. 2017; 37(7):790-801.

22. Paulus JK, Shah ND, Kent DM. All else being equal, men and women are still not the same: using risk models to understand gender disparities in care. Circ Cardiovasc Qual Outcomes. 2015;8(3):317-20.

23. Dahabreh IJ, Kent DM. Index event bias as an explanation for the paradoxes of recurrence risk research. JAMA. 2011:305:822-3.

\section{Submit your next manuscript to BioMed Central and we will help you at every step:}

- We accept pre-submission inquiries

- Our selector tool helps you to find the most relevant journal

- We provide round the clock customer support

- Convenient online submission

- Thorough peer review

- Inclusion in PubMed and all major indexing services

- Maximum visibility for your research

Submit your manuscript at www.biomedcentral.com/submit
Biomed Central 